\title{
Angiogenesis and bleeding disorders in FNAIT
}

Editorial

\section{Issaka Yougbare, Darko Zdravic and Heyu Ni}

Angiogenesis is a physiological process essential for embryo/fetal growth, wound healing, and repair of the myocardium after myocardial infarction. Angiogenesis is also implicated in pathological processes such as retinopathy and tumor growth. Anti-angiogenic agents have been demonstrated to have promising therapeutic potential for inhibiting tumour growth. Earlier studies suggested that $\beta 3$ integrin (i.e. $\alpha \mathrm{V} \beta 3$ that is formed by $\beta 3$ subunit and $\alpha \mathrm{V}$ subunit) expressed on angiogenic endothelial cells (ECs), is required for angiogenesis [1]; however, subsequent studies demonstrated that $\beta 3$ integrin deficiency $\left(\beta 3^{-/}\right)$did not prevent embryo/fetal growth and interestingly, enhanced pathological angiogenesis was observed in $\beta 3^{-/-}$mice [2]. It is therefore important to further study the roles of $\beta 3$ integrin in angiogenesis, fetal development and related diseases.

Fetal and neonatal alloimmune thrombocytopenia (FNAIT) is a life-threatening bleeding disorder that occurs when maternal alloantibodies cross the placenta and target paternally derived antigens, especially GPIIbIIIa (integrin $\alpha \operatorname{Ilb} \beta 3$ ) and GPIb $\alpha$, on fetal/neonatal platelets. In contrast to $\alpha \mathrm{V} \beta 3$, integrin $\alpha \operatorname{IIb} \beta 3$ is composed of a $\beta 3$ subunit and $\alpha \mathrm{IIb}$ subunit, which are almost exclusively expressed on platelets and megakaryocytes. Half of the polymorphisms known to cause FNAIT are located on the $\beta 3$ subunit. Approximately $80-90 \%$ of reported FNAIT cases are caused by antibodies targeting Human-PlateletAntigen-1a (HPA-1a) on $\beta 3$ subunit. Antibodies targeting the HPA-2a on GPIb $\alpha$ have also been reported. Intracranial hemorrhage (ICH), which occurs in $10-20 \%$ of affected fetuses/neonates, is a major clinical complication of FNAIT, leading to neurological impairment and death. Unfortunately, the mechanism responsible for $\mathrm{ICH}$, has only been inferred but not adequately explored.

Thrombocytopenia was considered to be the cause of bleeding in FNAIT. Interestingly, mice deficient in transcription factor NF-E2, which lack circulating platelets, did not develop significant bleeding disorders in utero. More strikingly, the murine fetuses with combined deficiencies in NF-E2 and fibrinogen genes exhibited normal embryonic development and were morphologically indistinguishable from their wild-type control counterparts at 18.5 days postcoitum [3]. Therefore, it is likely that neither thrombocytopenia nor blood coagulation are crucial for the development of ICH in FNAIT. Furthermore, several animal models have demonstrated that impairment of angiogenesis may contribute to bleeding in fetuses, particularly in their brains, since the brain is one of the major sites of angiogenesis during fetal development. However, whether ICH in FNAIT is caused by impairment of angiogenesis induced by maternal anti-platelet antibodies, is unclear. This is particularly interesting since the roles of $\beta 3$ integrin in angiogenesis are still controversial.

In a recent publication [4], we reported both active and passive murine models of FNAIT using $\beta 3^{-1-}$ and GPIb $\alpha^{-/-}$mice, in which gene deficient female mice were bred with wild-type males to mimic human FNAIT. We found that both maternal anti- $\beta 3$ and anti-GPIb $\alpha$ antibodies caused severe thrombocytopenia, however, ICH was only observed in anti- $\beta 3$-mediated FNAIT fetuses/ neonates (active model), and only anti- $\beta 3$ sera-injected neonates (passive model) developed ICH. Moreover, we found that anti- $\beta 3$ but not anti-GPIb $\alpha$ antibodies induced EC apoptosis, decreased the vessel density in the brains, and impaired the retinal vascular development. The anti- $\beta 3$ antibodies also decreased the expression of several pro-angiogenic factors, increased the expression of anti-angiogenic factors, and down-regulated Akt signalling. The antibodies also inhibited proliferation and network formation of human umbilical vein endothelial cells (HUVECs) in vitro. Importantly, anti-HPA-1a IgG purified from mothers who gave birth to neonates with FNAIT, also showed similar effects on HUVECs in vitro. This study suggests that anti-platelet $\beta 3$ antibodies also cross-react with $\alpha \mathrm{V} \beta 3$ on ECs, thereby impairing angiogenesis and contributing to ICH. The impairment of retinal vascular development may explain why some FNAIT untreated siblings suffered visual impairment in adulthood. Our study may also provide insights into the mechanisms of intrauterine growth restriction (IUGR) in FNAIT as well as the possible pathogenic role of $\alpha \mathrm{V} \beta 3$ integrin in tumorigenesis.

Clinical management of such devastating bleeding diatheses in fetuses/neonate remains a formidable challenge. Since our findings indicate that impairment of angiogenesis but not thrombocytopenia may be the major cause of ICH, invasive fetal platelet transfusion may not be an effective prenatal therapy. Our data demonstrated that antenatal administration of intravenous immunoglobulin (IVIG) decreased EC apoptosis, rescued brain and retinal vessel development, increased platelet counts, and ameliorated ICH, suggesting that IVIG may be an effective therapy [4]. In addition, our previous studies demonstrated that fetal (but not maternal) neonatal $\mathrm{Fc}$ receptor $(\mathrm{FcRn})$ is required for IgG trans-placental transport [6-7]. Whether 
anti-FcRn is a more effective and economical therapy for FNAIT requires further clinical studies.

Aside from its expression on platelets and ECs, $\beta 3$ integrin is also expressed on highly invasive trophoblasts, which are essential for normal placental vascular remodeling and development. Therefore, further studies on the effects of anti- $\beta 3$ antibodies on trophoblast proliferation, differentiation, migration, and invasion, may provide insights into the mechanism of IUGR and miscarriage in FNAIT, as well as aid in the development of new therapies to manage these disorders.

Heyu Ni: Toronto Platelet Immunobiology Group, Canadian Blood Services and Department of Laboratory Medicine and Pathobiology, Keenan Research Centre for Biomedical Science, St. Michael's Hospital, University of Toronto, Toronto, ON, Canada

Correspondence to: Heyu Ni, email nih@smh.ca

Received: June 17, 2015

Published: July 03, 2015

\section{REFERENCES}

1. Brooks, P.C. et al. Cell, 1994; 79:1157-1164.

2. Reynolds L.E. et al. Nat Med. 2002; 8:27-34.

3. Palumbo J.S. et al. 2004; J Thromb Haemost 2:1368-1379.4.

4. Yougbaré I. et al. J Clin Invest. 2015; 125:1545-56.

5. Ni H. et al. Blood. 2006; 107:2976-2983.

6. Chen P. et al. Blood. 2010; 116:3660-3668.

7. $\quad$ Li C. et al. J Clin Invest. 2011; 121:4537-4547. 